\title{
Altered Expression of 14-3-3 Genes in the Prefrontal Cortex of Subjects with Schizophrenia
}

\author{
Frank A Middleton ${ }^{1,2}$, Lansha Peng ${ }^{3}$, David A Lewis ${ }^{3,4}$, Pat Levitt ${ }^{3,5}$ and Karoly Mirnics*,1,3 \\ 'Department of Neurobiology, University of Pittsburgh School of Medicine, Pittsburgh, PA, USA; ' Department of Neuroscience \& Physiology, State \\ University of New York, Upstate Medical University, Syracuse, NY, USA; ${ }^{3}$ Department of Psychiatry, University of Pittsburgh School of Medicine, \\ Pittsburgh, PA, USA; ${ }^{4}$ Department of Neuroscience, University of Pittsburgh School of Medicine, Pittsburgh, PA, USA; ${ }^{5}$ John F Kennedy Center for \\ Human Development and Department of Pharmacology, Vanderbilt University School of Medicine, Nashville, TN, USA
}

\begin{abstract}
Seven distinct 14-3-3 proteins are expressed in mammals. One of the 14-3-3 genes (eta) has been previously associated with decreased expression in the prefrontal cortex (PFC) of subjects with schizophrenia. DNA microarray analysis of the PFC of 10 subjects with schizophrenia and 10 matched controls indicated that the majority of 14-3-3 genes exhibited moderate to marked decreases in expression in schizophrenia, which were significant at the group level across all 10 comparisons $(p<0.021)$. Selected changes in gene expression were further examined using in situ hybridization (ISH) in the same subject pairs as well as in four monkeys treated chronically with haloperidol and matched control animals. All analyses were performed blind to subject identity and diagnosis, or treatment. ISH analysis and multivariate analysis of covariance confirmed the significant decreases in expression of two I4-3-3 genes: beta - 31.9\%, zeta -18.2\%. Two other 14-3-3 genes exhibited more modest decreases in expression levels that were significant only in pairwise comparisons that did not factor in post-mortem interval or tissue storage time: gamma $-11.9 \%$, eta $-15.4 \%$. In the PFC of haloperidoltreated monkeys, there was no difference in 14-3-3 zeta expression, while 14-3-3 beta increased $28 \%(p<0.05)$ as a result of neuroleptic treatment. Our results suggest that decreased expression of selected 14-3-3 genes is a common feature of schizophrenia and that the 14-3-3 beta transcript may be unique among the 14-3-3 genes in its increase in response to haloperidol and decrease in the disease state. Neuropsychopharmacology (2005) 30, 974-983, advance online publication, 23 February 2005; doi: I 0.1 038/sj.npp. I 300674
\end{abstract}

Keywords: DNA microarray; schizophrenia; haloperidol; prefrontal cortex; in situ hybridization; gene expression

\section{INTRODUCTION}

The 14-3-3 proteins have been implicated in several recent studies exploring the potential genetic bases of schizophrenia (Bell et al, 2000; Toyooka et al, 1999; Vawter et al, 2001; Wong et al, 2003). Seven distinct 14-3-3 proteins are expressed in mammals (beta, eta, epsilon, gamma, sigma, theta, and zeta). This group of proteins plays an integral role in regulating many aspects of cellular function, including signal transduction, neurotransmitter metabolism, mitochondrial function, and cell cycle kinetics (Aitken et al, 1992; Fu et al, 2000; Morrison, 1994; Muslin and Xing, 2000; Skoulakis and Davis, 1998; Takahashi, 2003; van Hemert et al, 2001). 14-3-3 proteins typically exert this influence by forming dimers that sequester two proteins in

*Correspondence: Dr K Mirnics, Department of Psychiatry, University of Pittsburgh School of Medicine, WI655 BST, 381I O'Hara St, Pittsburgh, PA 15213, USA, Tel: + I 412648 9788, Fax: + I 412 6249910, E-mail: karoly+@pitt.edu

Received 25 October 2004; revised 30 November 2004; accepted 7 December 2004

Online publication: 17 December 2004 at http://www.acnp.org/citations/ NPPI $21704040504 /$ default.pdf close proximity in adjacent binding domains (see Aitken et al, 2002). This action generally enhances the activation of one of the proteins, but occasionally results in inhibition. To date, more than 100 different potential binding partners for 14-3-3 proteins have been identified. Interestingly, despite some evidence of distinct roles for certain 14-3-3 proteins, there also appears to be great potential for functional redundance of these proteins, since they can form both homo- and heterodimers.

In schizophrenia, mRNA levels of one of the 14-3-3 members (eta) has been reported to be significantly decreased in post-mortem cerebellum samples obtained from medicated subjects with schizophrenia (Vawter et al, 2001). This particular gene (and 14-3-3 theta as well) is located in the velocardial facial syndrome (VCFS)/schizophrenia susceptibility region (22q12-q13). In addition, significant association of specific tandem repeats in the 5'UTR of this gene has been reported in one study of Japanese subjects (Toyooka et al, 1999) and one study of subjects of Portuguese descent (Wong et al, 2003), although not in another study in the UK (Bell et al, 2000).

In our previous microarray studies of prefrontal cortical gene expression in schizophrenia, we reported that certain 
groups of genes regulating presynaptic, postsynaptic, and metabolic functions were consistently and significantly decreased in schizophrenia (Middleton et al, 2002; Mirnics et al, 2000). These gene groups represent a set of functionally defined intracellular cascades. In the present study, we examined the same data set to determine whether the family of 14-3-3 genes showed expression abnormalities in schizophrenia.

Gene families (as opposed to functional gene groups) are composed of individual transcripts that generally have a high degree of sequence similarity. Thus, it is possible that any group effect in the 14-3-3 gene family in our microarray data might be influenced by nonspecific (crosshybridization) events. Thus, we also sought to examine 14-3-3 gene expression patterns using independent techniques.

In the present study, our aims were to (1) use microarray data to examine the 14-3-3 gene family in the prefrontal cortex (PFC) of 10 pairs of subjects with schizophrenia and their matched controls; (2) determine at the group level, what type of relationship exists between the 14-3-3 gene family effect and previously reported alterations in functional gene groups in the same cohort of subjects; (3) verify the expression of each of the seven members of the 14-3-3 gene family in the human PFC using RT-PCR; (4) verify the schizophrenia-related changes in gene expression of selected members of the 14-3-3 gene family using highly specific riboprobes for quantitative in situ hybridization (ISH); and (5) examine the potential influence of antipsychotic medication on 14-3-3 gene expression.

\section{MATERIALS AND METHODS}

\section{Subjects}

The subjects and case-control pairings used for these studies are the same as those in a previous report (Middleton et al, 2002). Briefly, tissue from 10 subjects with schizophrenia and 11 matched control subjects was used for both the microarray and ISH studies (Table 1). One of the subject pairs (794c/665s) used in the microarray studies did not have tissue available for ISH from the control subject, so another matched control subject (806c) was substituted. The two groups of normal subjects and subjects with schizophrenia did not differ in mean $( \pm S D)$ age at the time of death $(47.3 \pm 14.5$ and $46.0 \pm 12.6$ years, respectively), post-mortem interval (PMI) (17.4 \pm 5.5 and $18.6 \pm 6.7 \mathrm{~h}$, respectively), brain $\mathrm{pH}(6.83 \pm 0.21$ and $6.84 \pm 0.35$, respectively), or tissue storage time at $-80^{\circ} \mathrm{C}$ $(57.7 \pm 16.6$ and $67.7 \pm 21.8$ months, respectively). Subject pairs were matched for gender (eight males and two females per group), and eight of the pairs were matched for race. Among the group of subjects diagnosed with schizophrenia, eight were receiving antipsychotic medications at the time of death, three had a history of alcohol abuse or dependence, and one had a history of drug dependence currently at the time of death. Two of the subjects with schizophrenia died by suicide. Among the control subjects, one $(635 c)$ had a past history of depressive disorder, not otherwise specified, and another had a history of alcohol abuse or dependence at the time of death. Consensus DSMIV diagnoses for all subjects were made using data from clinical records, toxicology studies, and structured interviews with surviving relatives, as described in detail previously (Volk et al, 2000).

\section{Microarray Experiments}

The explored microarray data set has been described previously (Middleton et al, 2002; Mirnics et al, 2000). While these previous manuscripts focused on presynaptic and metabolic changes associated with schizophrenia, the microarray data set also contained other promising leads, including expression changes in the transcripts encoding the $14-3-3$ protein family members. Owing to the structural, biochemical complexity of the 14-3-3 family of proteins and

Table I Characteristics of Subjects Used in Microarray and ISH Studies

\begin{tabular}{|c|c|c|c|c|c|c|c|c|c|c|c|c|}
\hline \multirow[b]{2}{*}{ Pair } & \multicolumn{2}{|c|}{ Gender } & \multicolumn{2}{|c|}{ Race } & \multicolumn{2}{|c|}{ Aqe } & \multicolumn{2}{|c|}{ PMI (h) } & \multicolumn{2}{|c|}{ Brain pH } & \multicolumn{2}{|c|}{ Storage (mo) } \\
\hline & C & $\mathbf{S}$ & C & $\mathbf{S}$ & C & $\mathbf{S}$ & C & $\mathbf{S}$ & C & $\mathbf{S}$ & C & $\mathbf{S}$ \\
\hline $55 \mid c / 625 s$ & M & M & $\mathrm{Ca}$ & AA & 61 & 49 & 16.4 & 23.5 & 6.63 & 7.32 & 72 & 60 \\
\hline $685 c / 622 s$ & M & M & $\mathrm{Ca}$ & $\mathrm{Ca}$ & 56 & 58 & 14.5 & 18.9 & 6.57 & 6.78 & 52 & 60 \\
\hline $806 c / 665 s$ & M & $M$ & $\mathrm{Ca}$ & $\mathrm{AA}$ & 57 & 59 & 24.0 & 28.1 & 6.94 & 6.92 & 31 & 55 \\
\hline $822 c / 787 s$ & M & $M$ & $\mathrm{AA}$ & AA & 28 & 27 & 25.3 & 19.2 & 7.04 & 6.67 & 28 & 35 \\
\hline $567 c / 537 s$ & $\mathrm{~F}$ & $\mathrm{~F}$ & $\mathrm{Ca}$ & $\mathrm{Ca}$ & 46 & 37 & 15.0 & 14.5 & 6.72 & 6.68 & 69 & 74 \\
\hline $516 c / 547 s$ & M & $M$ & AA & AA & 20 & 27 & 14.0 & 16.5 & 6.86 & 6.95 & 76 & 72 \\
\hline $630 c / 566 s$ & M & M & $\mathrm{Ca}$ & $\mathrm{Ca}$ & 65 & 63 & 21.2 & 18.3 & 6.95 & 6.80 & 59 & 69 \\
\hline
\end{tabular}

$\mathrm{C}=$ control subject; $\mathrm{S}=$ schizophrenic subject; $\mathrm{M}=$ male; $\mathrm{F}=$ female; $\mathrm{Ca}=$ Caucasian; $\mathrm{AA}=$ African American; PMI = post-mortem interval; mo = months. 
their unknown distribution in the human cortex, a comprehensive follow-up was essential to validate the specificity of the observed changes in the 14-3-3 transcript family.

Methods of tissue preparation, nucleic acid isolation, sample labeling, microarray hybridization and initial data analysis were the same as those reported previously (Middleton et al, 2002; Mirnics et al, 2000). Briefly, $200 \mathrm{ng}$ of mRNA was reverse transcribed using Cy3- or Cy5-labeled fluorescent primers. Samples from matched subject pairs were combined and hybridized onto the same UniGEM V or UniGEM V2 cDNA microarray (Incyte Genomics Inc., Fremont, CA). Each UniGEM V array contained over 7800 unique and sequence verified cDNA or EST elements, while each UniGEM V2 array contained nearly 10000 elements, including more than 7000 of the genes present on the UniGEM V. If a transcript was differentially expressed, the cDNA feature on the array bound more of the labeled target from one sample than the other, producing either a greater Cy3 or Cy5 signal intensity. Microarrays were scanned under $\mathrm{Cy} 3-\mathrm{Cy} 5$ dual fluorescence, and the resulting images were analyzed for signal intensity. Only genes whose signal intensity was 3.5-fold greater than background-signal intensity were called present. The operators performing the labeling, hybridization, scanning, and signal analysis were blind to the diagnostic category to which each sample belonged.

Owing to the inherent variability in the distribution of expression ratios from experiment to experiment, and the use of two different microarray platforms with different published confidence levels, we converted the balanced differential expression (BDE) ratio (of $\mathrm{Cy} 3: \mathrm{Cy} 5$ intensity) for each gene into a standard $Z$ score for each gene as follows:

$$
Z=\frac{\text { individual gene } \mathrm{BDE}-\text { mean } \mathrm{BDE} \text { of each array }}{\text { standard deviation of mean } \mathrm{BDE}}
$$

After this normalization procedure, the mean $Z$ score for all genes on each array comparison was 0.0 , with a standard deviation of 1.0 .

The 14-3-3 gene family members represented on the microarrays included six of the seven known 14-3-3 genes (beta, eta, epsilon, sigma, theta, and zeta), but did not include 14-3-3 gamma. The metabolic and other functionally defined gene groups discussed in this report were constructed as described previously (Middleton et al, 2002; Mirnics et al, 2000) using the Kyoto Encyclopedia of Genes and Genomes (KEGG) Release 19.0, July 2001 (web site: http://www.genome.ad.jp/kegg/metabolism.html).

Analysis of gene group expression was performed by analysis of variance (ANOVA), using a post hoc test (Scheffe) to compare the distribution of $Z$ scores for all genes in a group to the distribution of $Z$ scores for all the genes on an array. The probability values ( $p$ values) of these post hoc tests were normalized by log transformation, with the sign positive or negative depending on the direction of the change in expression. A correlation matrix was then computed to probe for correlated effects occurring among the 14-3-3 gene family group and 72 other functional gene groups that were described in previous papers (Middleton et al, 2002; Mirnics et al, 2000). For this analysis, varimax principal component analysis (PCA) was used. The factor loadings for the 14-3-3 gene family group, presynaptic gene group, and five gene metabolic groups described as significantly changed in five or more comparisons from previous studies were displayed in a radial plot (Figure 1, bottom).

\section{ISH Analysis}

Tissue blocks. The same tissue blocks used for the microarray experiments were used to obtain sections for ISH. Area 9 was identified based on surface landmarks, as previously described, and confirmed by examination of Nissl-stained sections (Glantz and Lewis, 1997). Sections $(20 \mu \mathrm{m})$ were cut with a cryostat at $-20^{\circ} \mathrm{C}$, mounted onto gelatin-coated glass slides, and stored at $-80^{\circ} \mathrm{C}$ until use. The slides were coded so that the investigator performing the analysis was blind to the diagnosis of the subjects. Three slides from each subject were used to examine the expression of each of the four different 14-3-3 genes: beta, gamma, eta, and zeta.

PCR, riboprobe generation, and specificity tests. Custom primers for each 14-3-3 gene were designed with Primer3 software (Supplemental Table 1). Approximately $500 \mathrm{ng}$ of total RNA from area 9 of a control subject was used to generate a template for PCR using reverse transcriptase (Superscript II, Gibco) and oligodT primer. After this step, approximately $10 \mathrm{ng}$ of double-stranded, first-strand template was used in a standard 35-cycle PCR reaction, using AmpliTaq Gold (PE Biosystems). Products were resolved on $2 \%$ agarose/ethidium bromide gels and each yielded a single band of the predicted size (data not shown). To generate the riboprobes for ISH, the specific products of these PCR reactions were cloned into a plasmid vector (pStBlue, Novagen) and transformed into competent Escherichia coli cells. After sequence verification of the cloned products, cloned inserts with T7 or SP6 RNA polymerase sites were amplified and used as a template for in vitro transcription (IVT). During IVT, ${ }^{35}$ S-labeled CTP was incorporated into each riboprobe. After purification of the labeled riboprobe and measurement of the specific activity of each probe, we performed ISH using approximately $2-3 \mathrm{ng}$ of probe $\left(\sim 1-2 \times 10^{6} \mathrm{DPM}\right)$ per slide in a total volume of $90-100 \mu \mathrm{l}$. All other methods used were described previously (Mirnics et al, 2000). Following hybridization $\left(16 \mathrm{~h}, 56^{\circ} \mathrm{C}\right)$ and film exposure (16-35 h, BioMax MR), high-resolution scans of each film image were used for quantification of signal with Scion Image (version 4.0b). In addition, darkfield images were captured from the slides that had been dipped in radiographic emulsion (Kodak NTB-2, 14 days). Through all procedures, subject pairs were always processed in parallel. Hybridization of sections with sense riboprobe did not result in detectable signal (data not shown). The absolute levels $\left(\mathrm{DPM} / \mathrm{mm}^{2}\right)$ of radioactive probe labeling were calculated using ${ }^{14} \mathrm{C}$-labeled standards that had been crosscalibrated to known quantities of $\left[{ }^{35} \mathrm{~S}\right]$-containing brain matter. The baseline levels for these measurements were set at the zero DPM level included on each standard.

To examine the specificity of each probe, we performed two types of analysis. First, using a Multiple Tissue Northern Blot (Clontech-BD), we examined the size of the 14-3-3 beta and epsilon transcripts across multiple brain 
a

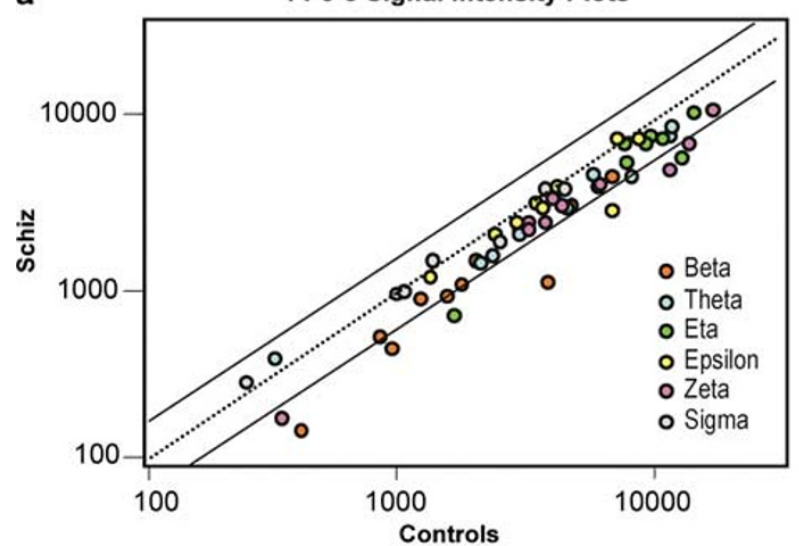

b

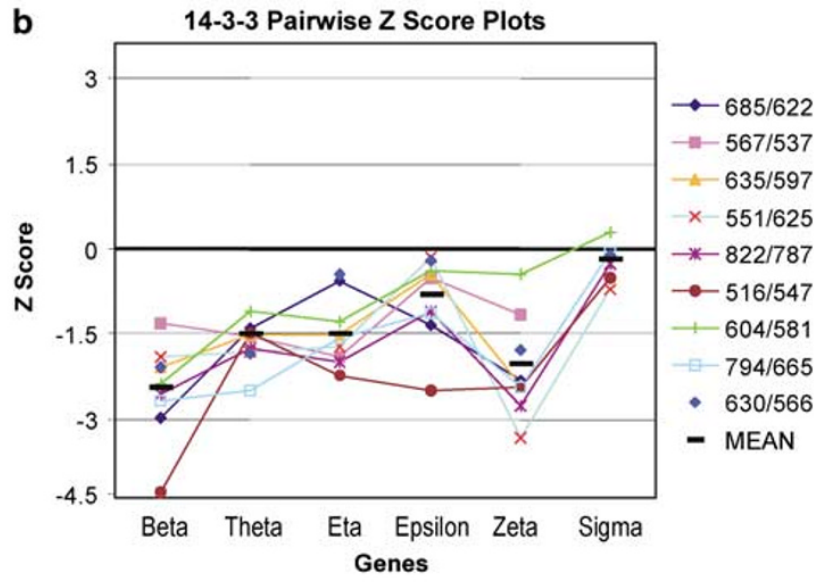

C

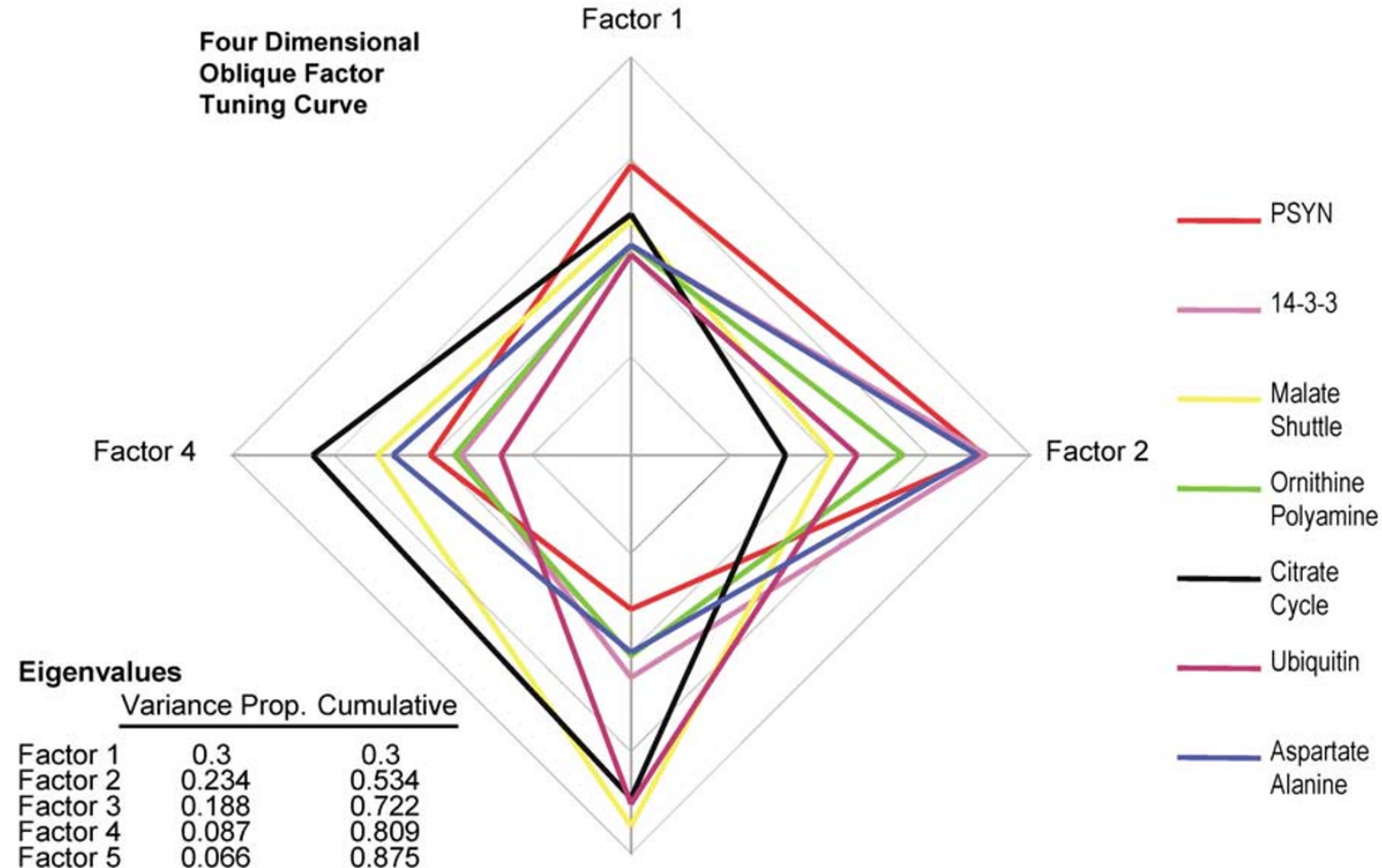

0.928

Factor $7 \quad 0.052 \quad 0.98$

Factor 3

Figure I Microarray analysis of I4-3-3 gene expression. The transcript levels of six I4-3-3 genes were examined using high-density cDNA microarrays on post-mortem samples of area 9 from 10 subjects with schizophrenia and their matched controls. (a) This analysis revealed a consistent shift toward reduced expression of most 14-3-3 transcripts. The fluorescent signal intensity for each matched subject pair is plotted on a log scale. The dashed line indicates equal expression, whereas the solid line indicates two-fold changes in expression. (b) The differences reported by the microarray were converted into $Z$ scores, which revealed that the most significantly affected transcripts were 14-3-3 beta and zeta, followed by eta and theta. The mean $Z$ score for each transcript is indicated by the solid bar. Notably, only the beta and zeta genes had mean $Z$ scores less than -1.96 (the two-tailed cutoff for significance) across the 10 subject pairs. (c) Using PCA, the effect on the 14-3-3 gene group was analyzed in relation to previously documented effects on selected metabolic gene groups and the presynaptic (PSYN) secretory function gene group. The factor weights for all of the significantly affected gene groups are displayed in a fourdimensional plot. Note that the 14-3-3, Aspartate/Alanine, and PSYN group effects appear to be highly correlated.

regions. This confirmed the presence of a single band in each brain area of the predicted size (Figure 2a). As all of the 14-3-3 transcripts are approximately the same size, we also examined potential crosshybridization using a customspotted membrane. In this case, $1 \mu \mathrm{l}(500 \mathrm{ng})$ of purified and freshly denatured, quick-frozen cDNA template was spotted onto a nitrocellulose membrane, UV crosslinked, and used for a membrane dot-blot protocol with ${ }^{32} \mathrm{P}$-labeled ribop- robes for selected 14-3-3 genes (Figure 2b). During the washing steps, all conditions were matched to those used in the ISH conditions.

To examine the potential of selection bias affecting our data collection, we also analyzed the within- and betweentissue block expression levels of three different 14-3-3 genes (14-3-3-beta, zeta, and eta). Four sections from each of the three superior frontal gyrus blocks containing area 9 were 

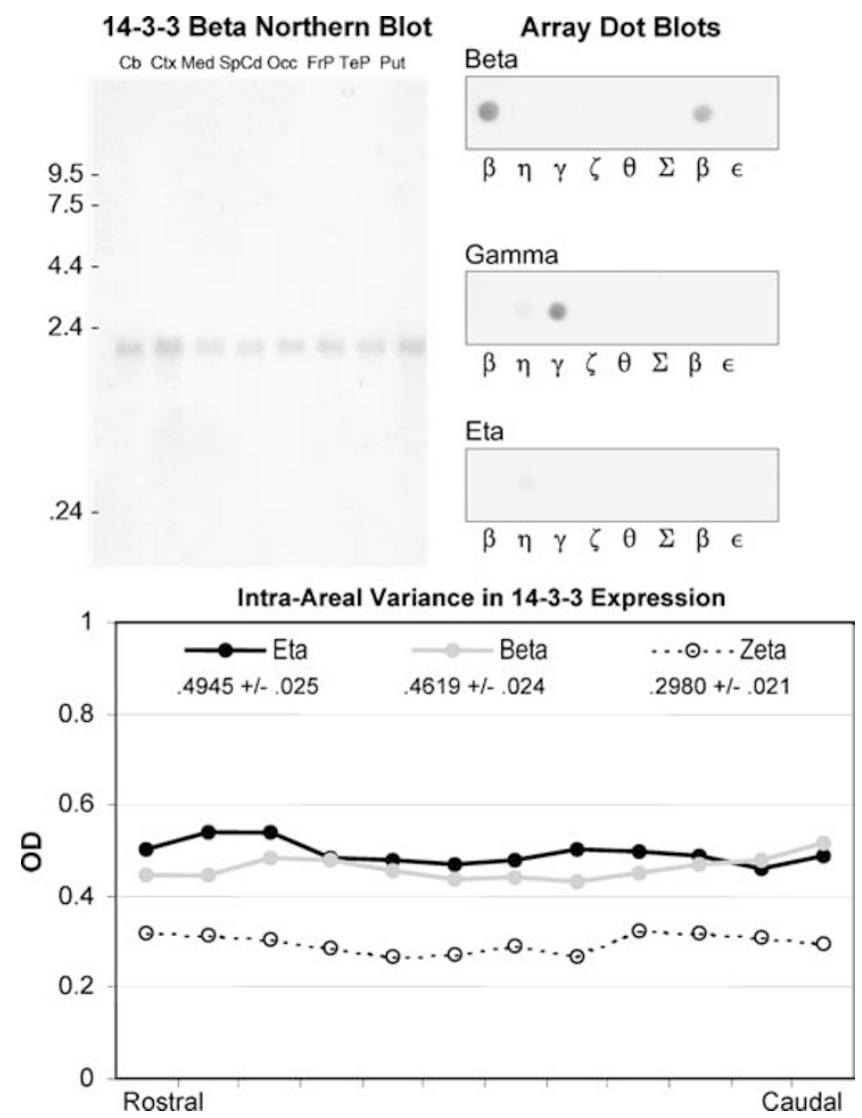

Figure 2 Examination of riboprobe specificity and intra-areal variability (Upper left) The expression of 14-3-3 beta and epsilon (not shown) was examined using a multiple tissue Northern blot. This revealed a single band across all brain regions examined. Nucleotide sizes are given at the left of the blot (in thousands). Cb, cerebellum; Ctx, cortex; Med, medulla; SpCd, spinal cord; Occ, occipital cortex; FrP, frontal pole; TeP, temporal pole; Put, putamen. (Upper right) The specificity of each riboprobe was further examined using custom cDNA dot blots. A measure of I $\mu$ l of cDNA from each 14-3-3 transcript was freshly denatured and spotted onto nitrocellulose membrane for hyridization with ${ }^{32} \mathrm{P}$-labeled cDNA probes corresponding to selected 14-3-3 genes. Images are from film exposed to the dot blots following washing. The location of the cDNA spot for each 14-3-3 transcript is given below the membrane (two different cDNAs for 14-3-3 beta were used). Note the high degree of specificity. (Bottom) The expression levels of three different 14-3-3 genes were examined in 12 sections from three adjacent tissue blocks from a single control subject using ${ }^{32}$ S-labeled riboprobes. The optical density of each section is plotted along the entire sampled zone. There was no apparent intra-areal (ie rostrocaudal) gradient within or between blocks. Values are mean \pm SE.

used for this analysis. Results indicated a fairly consistent pattern of OD signal intensity (Figure 2c) for each of the genes, with the standard error representing no more than $7 \%$ of the OD signal for any given gene, and interblock variability being no greater than intrablock variability.

Riboprobe and ISH data analysis. Crosshybridization data were analyzed using exploratory correlation analysis to generate estimates of the crosshybridization signal. In most cases, these estimates indicated that at least $85 \%$ of the signal being measured was coming from the intended target. Data from the ISH experiments were analyzed using multivariate analysis of covariance (MANCOVA) for all four 14-3-3 genes with diagnosis as the main effect, and PMI and tissue storage time as covariates. These models were applied both with and without subject pair as a blocking factor. Post hoc tests of significant main effects were performed using Scheffe's method. In addition, we also examined potential confounds between tissue and subject variables using exploratory correlation analysis.

Monkey experiments. To examine formally the potential influence of antipsychotic medication on the expression of the most robustly affected 14-3-3 genes (14-3-3 beta and zeta), we also used four pairs of male cynomolgus (Macaca fascicularis) monkeys, matched for age and weight, as subjects for ISH analysis in areas 9 and 46 . In each pair, one animal was treated for 9-12 months with the antipsychotic medication haloperidol decanoate, as described previously (Pierri et al, 1999). Serum levels were in the therapeutic range for the treatment of schizophrenia. Extrapyramidal symptoms were effectively managed by maintenance administration of benztropine mesylate. Tissue sections from these animals were acquired and used in parallel with the human material.

All procedures were reviewed and approved by the University of Pittsburgh's Institutional Review Board for Biomedical Research and Institutional Animal Care and Use Committee.

\section{RESULTS}

\section{4-3-3 Expression in Schizophrenia}

We examined the expression levels of all seven 14-3-3 genes in the PFC of 10 subjects with schizophrenia and their matched controls (Table 1) using either cDNA microarrays or ISH. According to the cDNA arrays, the majority of 14-33 genes exhibited moderate to severe decreases in expression in schizophrenia, which were significant at the level of the entire family across all 10 comparisons $(p<0.021)$ (Figure 1, Table 2).

\section{Relationship of 14-3-3 Group Effect and Previous Group Effects}

Having established that the expression of at least some 14-33 genes was significantly altered in subjects with schizophrenia, we wished to determine whether this family effect was related to the presynaptic and metabolic gene group effects we reported previously (see (Middleton et al, 2002; Mirnics et al, 2000). To carry out this analysis, we normalized the $p$-values (by log transformation) that were obtained by the gene group analyses as described previously (Middleton et al, 2002), and computed a correlation matrix between the 14-3-3 gene group effect and effects on 72 other gene groups that were described in previous papers (Middleton et al, 2002; Mirnics et al, 2000). Varimax PCA was then performed on this matrix, and the factor loading scores for each of the gene groups of interest displayed in a radial factor loading plot (Figure 1, bottom). This analysis indicated that the 14-3-3 gene group effect was most correlated with the presynaptic gene group (PSYN) and Aspartate/Alanine gene group (Asp/Ala). The similarities among these groups were evident in at least three of the four eigenvector dimensions. 
Table 2 Mean Pairwise Z Scores in 14-3-3 Gene Expression in Subjects with Schizophrenia

\begin{tabular}{|c|c|c|c|c|c|c|c|c|c|c|c|}
\hline Isoform & $685 / 622$ & $567 / 537$ & $635 / 597$ & $551 / 625$ & $822 / 787$ & $516 / 547$ & $604 / 581$ & $558 / 317$ & $794 / 665$ & $630 / 566$ & Mean \\
\hline Beta & -2.99 & -1.31 & -2.10 & -1.92 & -2.55 & -4.29 & -2.39 & -2.03 & -2.68 & -2.07 & -2.43 \\
\hline Theta & -1.41 & -1.54 & -1.52 & -1.81 & -1.77 & -1.48 & -1.12 & 0.10 & -2.49 & -1.85 & -1.49 \\
\hline Eta & -0.57 & -1.89 & $-|.5|$ & -1.73 & -2.01 & -2.23 & -1.28 & -1.76 & -1.58 & -0.45 & -1.50 \\
\hline Epsilon & -1.33 & -0.51 & -0.44 & -0.14 & -1.11 & -2.51 & -0.40 & -0.32 & -1.12 & -0.20 & -0.81 \\
\hline Zeta & -2.32 & -1.16 & -2.42 & -3.34 & -2.78 & -2.45 & -0.46 & -1.17 & -2.45 & -1.80 & -2.03 \\
\hline Mean & -1.72 & -1.28 & -1.60 & -1.61 & -1.75 & -2.25 & -0.89 & -0.86 & -1.73 & -1.08 & -1.47 \\
\hline$p$-value & 0.0059 & 0.0003 & 0.0021 & $<0.000 \mid$ & $<0.000 \mid$ & $<0.000 \mid$ & 0.0018 & 0.0208 & $<0.000 \mid$ & 0.005 & $<.021$ \\
\hline
\end{tabular}

\section{Expression by PCR}

Using PCR, we confirmed the robust expression of all seven 14-3-3 genes in area 9 of the human PFC (data not shown). Each of the 14-3-3 gene products amplified by PCR was visualized as a single bright band of the predicted size on an ethidium bromide-stained agarose gel. Although most of the 14-3-3 genes were highly expressed in the brain, 14-3-3 sigma appeared to be less abundant by this analysis. However, as we had virtually no data available on the cellular distribution of the 14-3-3 isoforms, for a meaningful interpretation of any gene expression changes, we needed to establish the anatomical distribution of the 14-3-3 family transcripts in the human cortex. As neither microarrays nor PCR could resolve the distribution of the 14-3-3 gene transcripts across the human cortical tissue, we needed to employ an independent validation method with a precise anatomical resolution. ISH was the method of choice in this regard: it could simultaneously provide a quantitative validation of gene expression changes and assess the anatomical distribution of the transcript in the studied tissue, yet it would not be dependent on putative tissue harvest biases, RNA isolation, reverse transcription, or amplification procedures.

\section{Probe Specificity}

Although it is possible to estimate potential crosshybridization signals using $\mathrm{Tm}$ formulae, we have found that the actual measures of crosshybridization can differ considerably from these calculations. To examine explicitly the specificity of each probe, we performed two types of analysis. First, using a multiple tissue Northern blot (Clontech-BD), we examined the size of the 14-3-3 beta and epsilon transcripts across multiple brain regions. This confirmed the presence of a single band in each brain area of the predicted size (Figure 2, top left). Thus, the 14-3-3 gene probes we cloned appeared to detect the correct size transcripts. However, because all of the 14-3-3 transcripts are approximately the same size, we also examined potential crosshybridization among 14-3-3 genes using a customspotted cDNA membrane. In this case, $1 \mu \mathrm{l}(0.5 \mathrm{ug})$ of each purified and freshly denatured, quick-frozen 14-3-3 cDNA was spotted onto a nitrocellulose membrane, UV crosslinked, and hybridized with ${ }^{32} \mathrm{P}$-labeled cDNA probes for selected 14-3-3 genes (Figure 2, top right). During the posthybridization washing steps, all conditions were matched to those used in the subsequent ISH conditions. The cDNA spot ODs were analyzed using exploratory correlation analysis to generate estimates of the crosshybridization signal. In most cases, these estimates indicated that at least $85 \%$ of the signal being measured was coming from the intended target.

\section{Intra-Areal Expression by ISH}

All ISH studies conducted with human post-mortem material must systematically and randomly sample the target area in order to produce unbiased results. It is usually assumed, but not empirically determined, that the sampling scheme is adequate enough to overcome potential intraareal variation in gene expression levels or patterns. To avoid the possibility of confounding our ISH analysis of 143-3 gene expression by sampling bias, we examined the variation in expression of three different 14-3-3 genes in three adjacent tissue blocks obtained from the superior frontal gyrus of a single control subject. This analysis involved four equally spaced sections from each of the three tissue blocks (12 sections per gene). Optical densities were determined for the entire section. This analysis revealed that the standard error of the OD measurement equalled approximately $5-7 \%$ of the total OD signal (Figure 2, bottom). Moreover, there did not appear to be any differences between the three blocks in overall expression for any of the three 14-3-3 genes examined.

\section{Examination of Differences in Expression by ISH}

Having established the specificity of each probe, and the lack of any apparent intra-areal effect, we next sought to confirm the patterns of expression differences reported by the microarrays for three of the 14-3-3 genes (beta, zeta, and eta) and also to examine the potential expression abnormalities in the single 14-3-3 gene that was not represented on the array (14-3-3 gamma). MANCOVA confirmed the significant decreased expression in schizophrenia of two genes: beta $-31.9 \%\left(\mathrm{~F}_{1,9}=5.74, p=0.048\right)$ and zeta $-18.2 \%$ $\left(\mathrm{F}_{1,9}=16.98, p=0.005\right)$ (Figure 3$)$. Only in an alternate MANOVA model that did not account for PMI and tissue storage time did the 14-3-3 eta and gamma transcripts show 

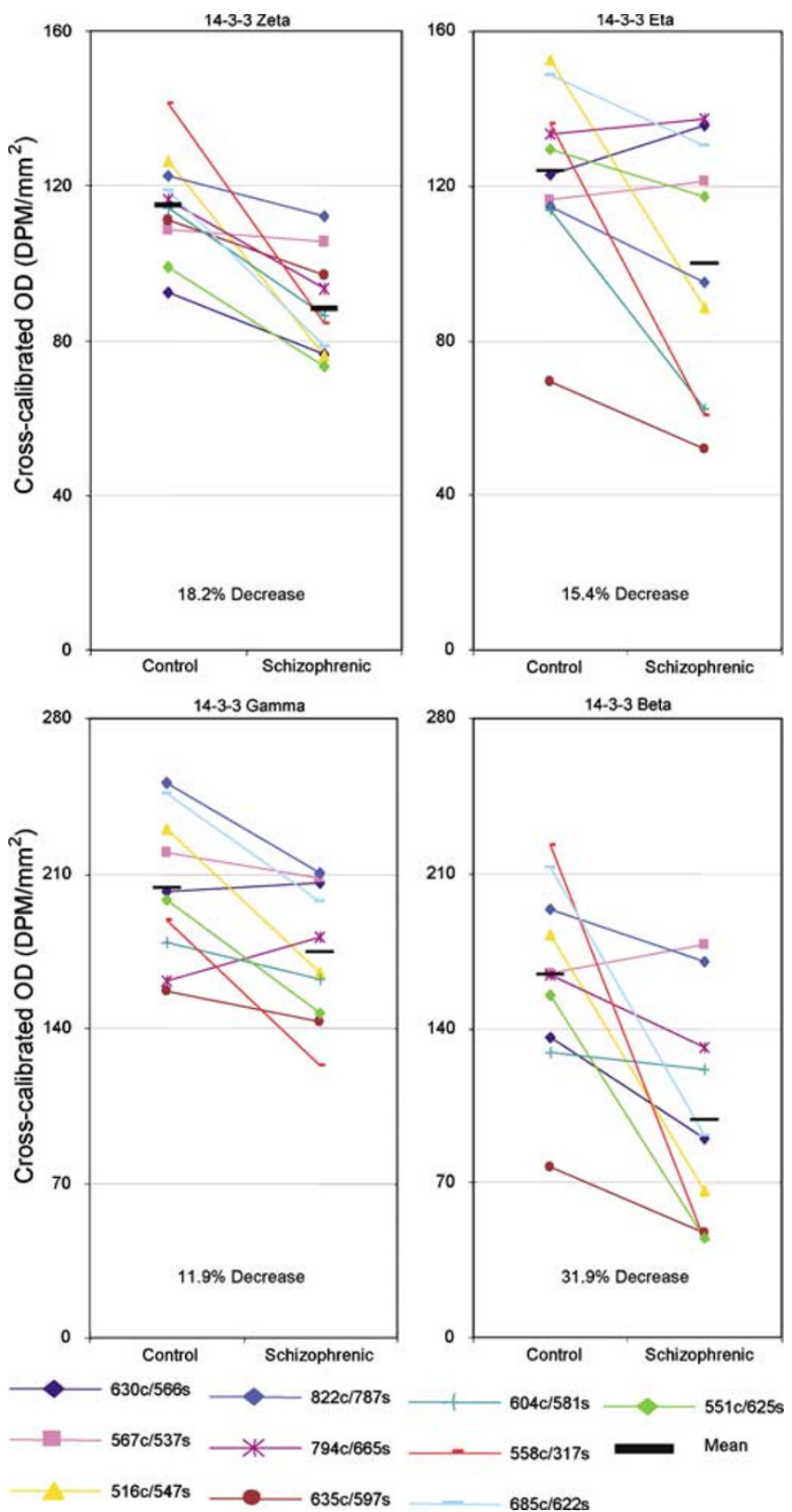

Figure 3 Examination of pairwise expression differences in 14-3-3 transcripts. The mean calibrated absolute intensity level (in disintegrations per minute per square millimeter of tissue) of four different 14-3-3 genes was obtained using three sections per subject through area 9 of the PFC. The mean expression for all 10 subjects in each group is given by the solid bar. Lines connect subject pairs. Differences were calculated using pairwise MANCOVA with diagnosis as the main effect and PMI as a covariate. We observed highly significant decreased expression of 14-3-3 beta and zeta, but only marginal decreases in eta and gamma.

significant pairwise decreases in expression $(-15.4$ and $-11.9 \%$, respectively). Sheffe's post hoc test confirmed the significance of the 14-3-3 beta and zeta findings ( $p=0.0094$ and 0.0007 , respectively).

\section{Laminar Expression Patterns and Differences}

Within the PFC, each of the 14-3-3 genes we examined was expressed predominately in the gray matter (Figure 4). In

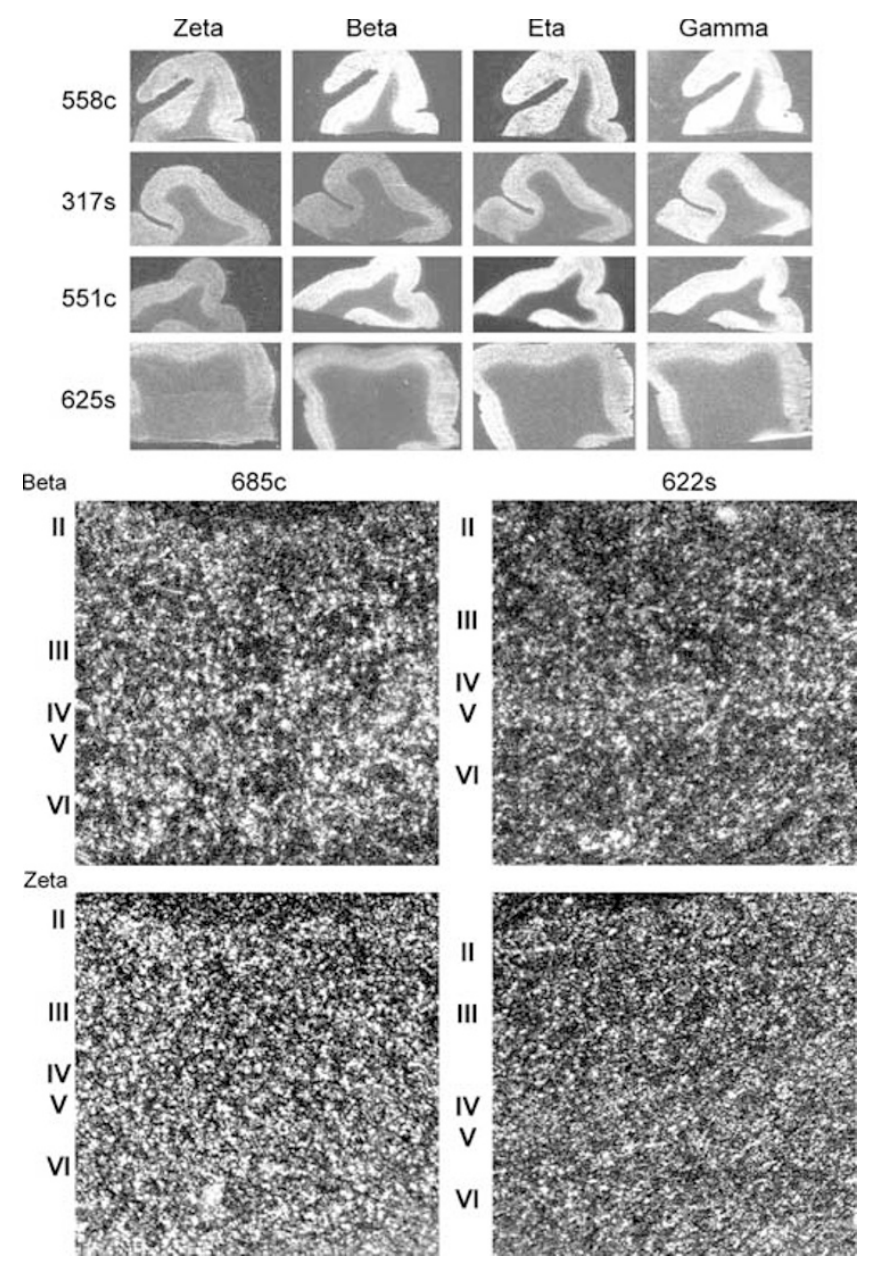

Figure 4 Patterns of prefrontal 14-3-3 gene expression and differences in expression. The transcripts for 14-3-3 beta and gamma were the most abundant according to ${ }^{35} \mathrm{~S}$-labeled riboprobe ISH analyses. Each image indicates the signal obtained (viewed in darkfield) from a single $20 \mu \mathrm{m}$ section through area 9 of the PFC. (Upper) Subject pairs represented include $558 \mathrm{c} / 317 \mathrm{~s}$ and $55 \mathrm{Ic} / 625 \mathrm{~s}$. (Lower) The expression of 14-3-3 beta and zeta is shown at high power for another subject pair $(685 \mathrm{c} / 622 \mathrm{~s})$. Note the loss of transcript expression throughout most cortical layers. c, control subject; s, subject with schizophrenia; roman numerals II-VI indicate cortical layers.

controls, the signals obtained for 14-3-3 gamma and beta were the highest, with eta and zeta less (Figures 3 and 4 ). Within the cortical lamina, the pairwise changes in expression appeared to be present throughout each layer, although in some cases there appeared to be a relative sparing of the layer $\mathrm{V}$ signal for 14-3-3 beta (Figure 4, bottom).

\section{Examination of Potential Confounding Factors}

Our probe specificity tests indicated that as much as $15 \%$ of the signal for any given gene could be derived from other 14-3-3 genes. This prompted us to examine whether there was a relationship between nucleotide similarity and the amount of OD signal obtained in the ISH experiments. A strong correlation could indicate crosshybridization contamination of our results, while a weak correlation would 
Table 3 (a) Correlations of In Situ Values and Tissue/Subject Variables and (b) Nucleotide Similarity of Riboprobes

\begin{tabular}{|c|c|c|c|c|c|c|c|}
\hline & Zeta & Eta & Gamma & Age & PMI & PH & Storage \\
\hline \multicolumn{8}{|c|}{ (a) Correlations of in situ values and tissue/subject variables } \\
\hline Beta & 0.801 & 0.621 & 0.77 & -0.206 & -0.043 & 0.01 & -0.37 \\
\hline Zeta & & 0.315 & 0.469 & -0.344 & -0.3 & -0.07 & -0.292 \\
\hline Eta & & & 0.66 & 0.146 & 0.079 & 0.11 & -0.276 \\
\hline Gamma & & & & -0.2 & 0.1 & 0.031 & -0.373 \\
\hline Age & & & & & 0.099 & -0.147 & 0.076 \\
\hline PMI & & & & & & 0.494 & -0.549 \\
\hline $\mathrm{pH}$ & & & & & & & -0.435 \\
\hline
\end{tabular}

Zeta Eta Gamma Sigma Theta Epsilon

(b) Nucleotide similarity of ribroprobes

$\begin{array}{lllllll}\text { Beta } & 64 & 67 & 16 & 73 & 63 & 62 \\ \text { Zeta } & & 69 & 24 & 67 & 64 & 72 \\ \text { Eta } & & & 31 & 75 & 70 & 71 \\ \text { Gamma } & & & 24 & 25 & 24 \\ \text { Sigma } & & & & 68 & 69 \\ \text { Theta } & & & & & 72\end{array}$

Epsilon

strengthen our results and suggest that, although some of the 14-3-3 genes might be expressed in a similar manner, these similarities are not due to crosshybridization. Our analysis indicated that fairly high correlations $(R=0.62-$ 0.8 ) were present between 14-3-3 beta and each of the other 14-3-3 genes (Table 3a). Likewise, 14-3-3 eta and gamma also exhibited some similarity in expression levels among the 20 subjects $(R=0.66)$. Interestingly, the similarities seen in the OD measurements did not correlate with the amount of nucleotide similarity between the same 14-3-3 genes (Table $3 \mathrm{~b} ; R=-0.25$ ). Thus, the amount of correlation seen in the different 14-3-3 genes would appear to be due to similarities in expression pattern among the genes, not due to crosshybridization events.

To examine the potential for antipsychotic medication to influence our results, we examined 14-3-3 gene expression in four PFC areas 9 and 46 from male cynomolgus monkeys treated chronically with haloperidol decanoate and benzotropine and matched control animals. We examined the expression pattern of the two most robustly affected 14-3-3 genes in the human studies (14-3-3 beta and zeta) in three to four sections from each animal. There was no difference in 14-3-3 zeta expression, while 14-3-3 beta increased $28 \%$ $(p<0.05$; Figure 5 , top $)$. This increase is in direct contrast to the decrease seen in the subjects with schizophrenia, and appeared to occur primarily in pyramidal cell layers (Figure 5, bottom).

\section{DISCUSSION}

Our microarray studies of the PFC in subjects with schizophrenia revealed three principal findings regarding
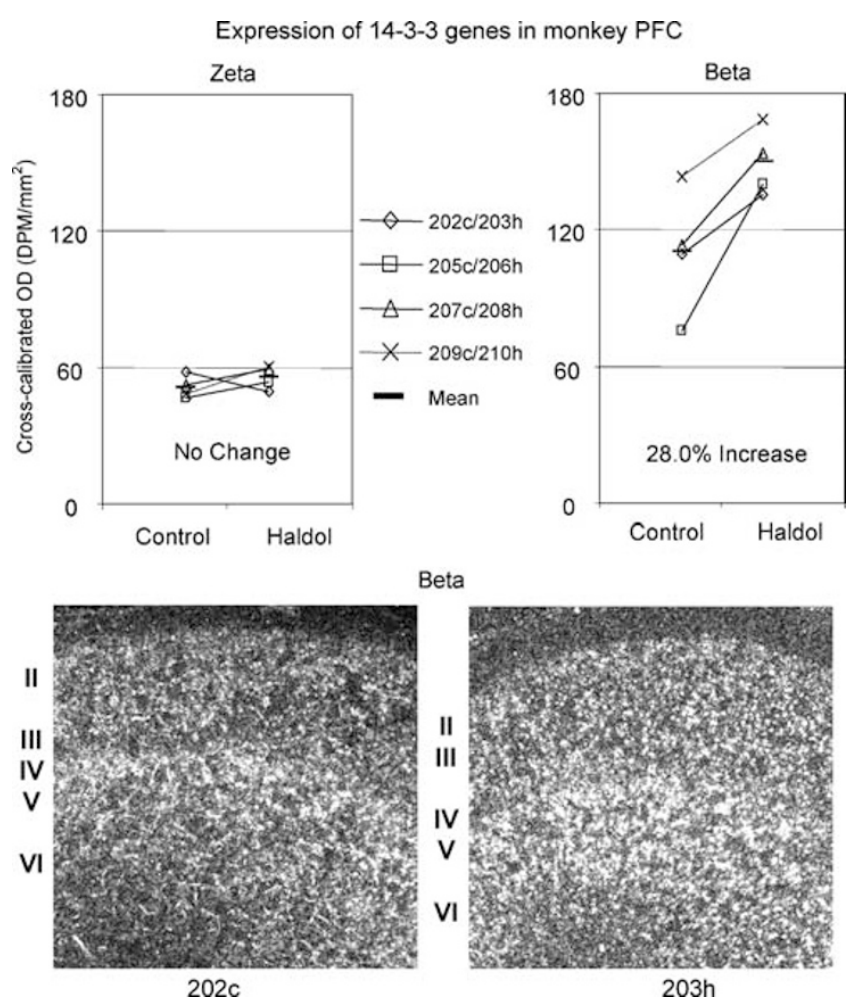

Figure 5 Examination of potential medication effects on 14-3-3 beta and zeta expression in monkey prefrontal cortex. The two significantly affected 14-3-3 genes (beta and zeta) were examined in a primate model of typical neuroleptic treatment (Pierri et al, 200 I). Expression was quantified in four pairs of animals using ${ }^{35} \mathrm{~S}$-labeled riboprobes on multiple sections per animal through area 9 of the prefrontal cortex. Units and conventions are the same as in Figure 3. The darkfield image at the bottom is from area 9 of a representative monkey pair, illustrating the large increase in pairwise expression for the 14-3-3 beta transcript. Note that this gene was significantly decreased in the subjects with schizophrenia.

the expression of 14-3-3 gene family members: (1) of the six 14-3-3 genes represented on the arrays (beta, eta, epsilon, sigma, theta, and zeta), five were detectable in all 10 pairwise comparisons, with one (sigma) detectable in $7 / 10$ comparisons; (2) all of the 14-3-3 family members except for sigma were decreased in most comparisons; (3) at the group level, these genes were significantly changed in all 10 arrays $(p<0.021)$, representing the single most-changed gene group we have identified to date. The expression changes reported on the microarrays were confirmed by $\mathrm{ISH}$, which revealed decreases for zeta and beta $>$ eta $>$ gamma, supporting the microarray findings.

Using factor analysis, we determined that the expression changes of all six 14-3-3 genes were moderately well correlated across all 10 subject pairs (eigenvalue $=3.09$, variance proportion $=0.515)$. Among the 14-3-3 genes, the theta transcript showed the weakest association (unrotated factor weight $=0.432$ ) compared to all the other 14-3-3 genes (variance proportion range $=0.713-0.825$ ). This indicates that the specific ranking of the changes in 14-3-3 genes across the subject pairs is somewhat consistent.

We have previously documented changes in the presynaptic and selected metabolic gene groups in schizophrenia. A direct comparison of all metabolic pathways, the presynaptic gene group, and the 14-3-3 gene family 
indicated a very high correlation for the expression changes in the 14-3-3 gene group with the presynaptic and Aspartate/Alanine metabolic (variance proportion $>0.75$ ) gene groups and a lower correlation with the Ornithine/ Polyamine metabolic gene group. The factor tuning curve (Figure 2) clearly shows that the relationship between these gene groups is tightly clustered through four eigenvectors.

In schizophrenia, the expression of one 14-3-3 member (eta) is significantly decreased in post-mortem cerebellum samples obtained from medicated schizophrenic subjects (Vawter et al, 2001). This particular gene (and 14-3-3 theta as well) is located in the VCFS/schizophrenia susceptibility region (22q12-q13), and significant association of certain tandem repeats in the $5^{\prime}$-UTR of this gene was found in one study of Japanese subjects (Toyooka et al, 1999) and sequence abnormalities in subjects of Portuguese descent (Wong et al, 2003), although not in a follow-up study in the UK (Bell et al, 2000). Collectively, our data and those of these groups strongly support the notion of there being a conserved alteration in 14-3-3 gene family expression and possibly even sequence alteration in subjects with schizophrenia. Our observations regarding the decreases in expression of 14-3-3 beta and zeta are novel, but are not likely to be directly related to the findings regarding the genetic risk for schizophrenia. The 14-3-3 beta is located at $20 \mathrm{q} 13.1$ and zeta is located at 2p25.2-p25.1. Neither one of these loci have any strong associations or linkages with schizophrenia. Nonetheless, the changes in these transcripts may form part of the conserved pathophysiology of the disease.

As the 14-3-3 family of proteins plays an integral role in regulating many aspects of cellular function in the brain, including signal transduction, neurotransmitter metabolism, and mitochondrial function (there are at least 100 different binding partners for these proteins that have been identified), it is difficult to precisely define the appropriate context in which to view 14-3-3 gene alterations in schizophrenia. Of the more than 300 functionally defined gene groups that we have studied in the same cohort of subjects, we find compelling evidence that the magnitude and statistical significance of the 14-3-3 gene family effect closely parallels (and is highly correlated with) the changes in presynaptic secretory function and Aspartate/Alanine metabolism gene groups (see Figure 1). Thus, we tentatively suggest that at least in schizophrenia, the most critical functions, that an effect of the 14-3-3 gene family might exert, are those related to neurotransmission and neurotransmitter metabolism. Indeed, the 14-3-3 gene familiy was originally characterized by the descriptive title of tyrosine monooxygenase-/tryptophan monooxygenase-activating proteins. The decreased synthesis of multiple 14-3-3 transcripts should produce in the cell a decrease in the amount of dopamine available for neurotransmission - an event that could influence both excitatory and inhibitory neurotransmission via D1 and D2 receptor classes. The decreased aspartate metabolism could reduce the amount of excitatory neurotransmitters (including both aspartate and glutamate) available for release, as well as the bioenergetic properties of mitochondria localized to the synapse. The decrease in the presynaptic secretory machinery group genes that also occurs in these subjects suggests that the substrates for neurotransmission as well as the machinery for neurotransmission are collectively and profoundly altered in schizophrenia.

Our observations of altered expression of the 14-3-3 gene group may also be of interest in relationship to the disturbances in markers of GABA neurotransmission in the PFC of subjects with schizophrenia. These include reductions in the $67 \mathrm{kDa}$ form of glutamatic acid decarboxylase, GABA membrane transporter and the calciumbinding protein parvalbumin in a subset of GABA neurons, including chandelier cells (Akbarian et al, 1995; Hashimoto et al, 2003; Volk et al, 2000, 2001), and an upregulation of GABA receptors containing the alpha2 subunit at the postsynaptic target of chandelier cells, the axon initial segment of pyramidal neurons (Volk et al, 2002). The 14-3-3 proteins (including the zeta and eta forms) have been shown to associate and colocalize with the $\mathrm{C}$-terminus of presynaptic $\mathrm{GABA}(\mathrm{B}) \mathrm{R} 1$ receptors in rat brain preparations and tissue cultured cells (Couve et al, 2001). Furthermore, in the presence of 14-3-3, the authors reported a reduction in the dimerization of $\mathrm{GABA}(\mathrm{B}) \mathrm{R} 1$ and $\mathrm{R} 2$ subunits. GABA(B)R1 is thought to account for the vast majority of GABA binding sites in the brain, but in the absence of $\mathrm{GABA}(\mathrm{B}) \mathrm{R} 2$, is poorly expressed on the plasma membrane and largely fails to couple to ion channels (Jones et al, 2000). The coupling of GABA(B)R2 to R1 permits surface expression of GABA(B)R1 and the appearance of potassium and calcium currents (Jones et al, 2000). Thus, it is possible that the reduction in 14-3-3 gene expression may represent a compensatory mechanism for overcoming a reduction in GABA signaling that is present in the PFC of subjects with schizophrenia.

Finally, we also consider the potential functional importance of our observations in light of the known interactions between 14-3-3 proteins and some of the proteins known as regulators of G-protein signaling (RGS) (Benzing et al, 2000). Although at this point there has been no evidence of a clear association between RGS4 and 14-3-3 proteins, we previously documented significant changes in one of the RGS proteins - RGS4 (Mirnics et al, 2001), in the same subjects used in the present study.

\section{ACKNOWLEDGEMENTS}

We thank Dr Takanori Hashimoto and Dianne Cruz for technical assistance, and members of the Conte Center for the Neuroscience of Mental Disorders for helpful comments and discussion. Financial support for this research was provided by a Young Investigator Award from the National Alliance for Research on Schizophrenia and Depression (FAM), Projects 1 (DAL) and 2 (PL, KM) of NIMH Center Grant MH45156 (DAL), and an endowment from the Richard King Mellon Foundation (PL).

\section{REFERENCES}

Aitken A, Amess B, Howell S, Jones D, Martin H, Patel Y et al (1992). The role of specific isoforms of 14-3-3 protein in regulating protein kinase activity in the brain. Biochem Soc Trans 20: 607-611.

Aitken A, Baxter H, Dubois T, Clokie S, Mackie S, Mitchell K et al (2002). Specificity of 14-3-3 isoform dimer interactions and phosphorylation. Biochem Soc Trans 30: 351-360. 
Akbarian S, Kim JJ, Potkin SG, Hagman JO, Tafazzoli A, Bunney Jr WE et al (1995). Gene expression for glutamic acid decarboxylase is reduced without loss of neurons in prefrontal cortex of schizophrenics. Arch Gen Psychiatry 52: 258-266.

Bell R, Munro J, Russ C, Powell JF, Bruinvels A, Kerwin RW et al (2000). Systematic screening of the 14-3-3 eta (eta) chain gene for polymorphic variants and case-control analysis in schizophrenia. Am J Med Genet 96: 736-743.

Benzing T, Yaffe MB, Arnould T, Sellin L, Schermer B, Schilling B et al (2000). 14-3-3 interacts with regulator of $G$ protein signaling proteins and modulates their activity. $J$ Biol Chem 275: 28167-28172.

Couve A, Kittler JT, Uren JM, Calver AR, Pangalos MN, Walsh FS et al (2001). Association of GABA(B) receptors and members of the 14-3-3 family of signaling proteins. Mol Cell Neurosci 17: $317-328$

Fu H, Subramanian RR, Masters SC (2000). 14-3-3 proteins: structure, function, and regulation. Annu Rev Pharmacol Toxicol 40: 617-647.

Glantz LA, Lewis DA (1997). Reduction of synaptophysin immunoreactivity in the prefrontal cortex of subjects with schizophrenia: regional and diagnostic specificity. Arch Gen Psychiatry 54: 943-952.

Hashimoto T, Volk DW, Eggan SM, Mirnics K, Pierri JN, Sun Z et al (2003). Gene expression deficits in a subclass of GABA neurons in the prefrontal cortex of subjects with schizophrenia. J Neurosci 23: 6315-6326.

Jones KA, Tamm JA, Craig DA, Yao W, Panico R (2000). Signal transduction by GABA(B) receptor heterodimers. Neuropsychopharmacology 23: S41-S49.

Middleton FA, Mirnics K, Pierri JN, Lewis DA, Levitt P (2002). Gene expression profiling reveals alterations of specific metabolic pathways in schizophrenia. J Neurosci 22: 2718-2729.

Mirnics K, Middleton FA, Marquez A, Lewis DA, Levitt P (2000). Molecular characterization of schizophrenia viewed by microarray analysis of gene expression in prefrontal cortex. Neuron 28: 53-67.

Mirnics K, Middleton FA, Stanwood GD, Lewis DA, Levitt P (2001). Disease-specific changes in regulator of G-protein signaling 4 (RGS4) expression in schizophrenia. Mol Psychiatry 6: 293-301.

Morrison D (1994). 14-3-3: modulators of signaling proteins? Science 266: 56-57.
Muslin AJ, Xing $\mathrm{H}$ (2000). 14-3-3 proteins: regulation of subcellular localization by molecular interference. Cell Signal 12: 703-709.

Pierri JN, Chaudry AS, Woo T-U, Lewis DA (1999). Decreased GAT-1-immunoreactive chandelier cell axon terminals in the prefrontal cortex in schizophrenia: diagnostic and laminar specificity. Schizophr Res 36: 83.

Pierri JN, Volk CLE, Auh S, Sampson A, Lewis DA (2001). Decreased somal size of deep layer 3 pyramidal neurons in the prefrontal cortex in subjects with schizophrenia. Arch Gen Psychiatry 58: 466-473.

Skoulakis EM, Davis RL (1998). 14-3-3 proteins in neuronal development and function. Mol Neurobiol 16: 269-284.

Takahashi Y (2003). The 14-3-3 proteins: gene, gene expression, and function. Neurochem Res 28: 1265-1273.

Toyooka K, Muratake T, Tanaka T, Igarashi S, Watanabe $\mathrm{H}$, Takeuchi $\mathrm{H}$ et al (1999). 14-3-3 protein eta chain gene (YWHAH) polymorphism and its genetic association with schizophrenia. Am J Med Genet 88: 164-167.

van Hemert MJ, Steensma HY, van Heusden GP (2001). 14-3-3 proteins: key regulators of cell division, signalling and apoptosis. Bioessays 23: 936-946.

Vawter MP, Barrett T, Cheadle C, Skolov BP, Wood III WH, Donovan DM et al (2001). Application of cDNA microarrays to examine gene expression differences in schizophrenia. Res Bull 55: 641-650.

Volk DW, Austin MC, Pierri JN, Sampson AR, Lewis DA (2000). Decreased $\mathrm{GAD}_{67}$ mRNA expression in a subset of prefrontal cortical GABA neurons in subjects with schizophrenia. Arch Gen Psychiatry 57: 237-245.

Volk DW, Austin MC, Pierri JN, Sampson AR, Lewis DA (2001). GABA transporter-1 mRNA in the prefrontal cortex in schizophrenia: decreased expression in a subset of neurons. Am J Psychiatry 158: 256-265.

Volk DW, Pierri JN, Fritschy J-M, Auh S, Sampson AR, Lewis DA (2002). Reciprocal alterations in pre- and postsynaptic inhibitory markers at chandelier cell inputs to pyramidal neurons in schizophrenia. Cereb Cortex 12: 1063-1070.

Wong AH, Macciardi F, Klempan T, Kawczynski W, Barr CL, Lakatoo S et al (2003). Identification of candidate genes for psychosis in rat models, and possible association between schizophrenia and the 14-3-3 eta gene. Mol Psychiatry 8: 156-166.

Supplementary Information accompanies the paper on Neuropsychopharmacology website (http://www.nature.com/npp) 